\title{
A tool for visually exploring multi-objective mixed-integer optimization models
}

\author{
Rui Borges Lopes ${ }^{1,2}$, Beatriz Sousa Santos ${ }^{3,4}$, Carlos Ferreira ${ }^{1,4}$ \\ ${ }^{1}$ Department of Economics, Management and Industrial Engineering, University of Aveiro \\ ${ }^{2}$ Center for Research \& Development in Mathematics and Applications, University of Aveiro \\ ${ }^{3}$ Department of Electronics Telecommunications and Informatics, University of Aveiro \\ ${ }^{4}$ Institute of Electronics Engineering and Telematics of Aveiro, University of Aveiro \\ \{rui.borges@ua.pt,bss@ua.pt,carlosf@ua.pt\}
}

\begin{abstract}
Multi-objective optimization models have been increasingly used as optimal decisions are searched in settings considering several conflicting objectives. In these cases compromises must be made and often a large number of nondominated optimal solutions exist. From these solutions decisionmakers must find the preferred one. This is a difficult task both from a computational and cognitive point of views, as it requires several solutions to be obtained and compared. An interactive visualization tool for fully understanding the best trade-offs is therefore becoming increasingly important. This paper proposes visualization solutions, implemented in a tool, for aiding decision-makers in finding the preferred solution in multiobjective optimization problems.
\end{abstract}

Keywords-multi-objective optimization; visual exploration tool.

\section{INTRODUCTION}

Mathematical models with integer variables have been used in many applications. Examples include modelling investment choices [1], production levels [2] and logistics decisions [3]. As most of these decisions are inherently multi-objective it is important to addressed them using multiobjective integer and mixed-integer programming (MOMIP). To deal with multi-objective problems, interactive methods have shown to be more effective as they enable to reduce the computational effort and usually provide means to assist the decision-maker (DM) in finding her/his preferred solution [4]. The preferred solution is typically obtained from the Pareto set and is the one chosen by the DM to implement. Note that Pareto sets cannot be computed efficiently in many cases; even if it is possible to find all non-dominated solutions exactly, they are often of exponential size [5].

In interactive methods there is an alternation between human intervention and computation phases. Preference elicitation (human intervention) and solution generation (computation) alternate until the DM considers having sufficient knowledge of the non-dominated (or Pareto) set. Iteratively, the DM is provided with some information and is asked to evaluate the proposed solutions, or to provide additional information regarding her/his preferences [6]. The underlying idea of interactive methods is the major motivation of contemporary decision support systems [7]. For a recent review on interactive methods for MOMIP the reader is referred to [8], and to [9] for concepts on multiobjective mixed-integer programming.

Recently, in [10], a new interactive method was proposed, extending the method by Ferreira et al. [11] to more than two objectives. However, the use of multi-objective models and methods often requires a lot of expertise. As most practitioners may lack this know-how, and a lot of information must be analyzed for each solution, this prompts the use of visualization and human-computer interaction methods. In this paper, several visualization solutions are presented for implementing the newly proposed interactive method in a tool. Section 2 outlines the method and Section 3 describes the proposed visualization solutions. In Section 4 results of a usability evaluation are presented and discussed, and section 5 concludes this paper.

\section{INTERACTIVE METHOD}

The interactive method tackles MOMIP problems by searching for non-dominated solutions in the objective's space (each having a representation in the decision's space - Fig. 1).

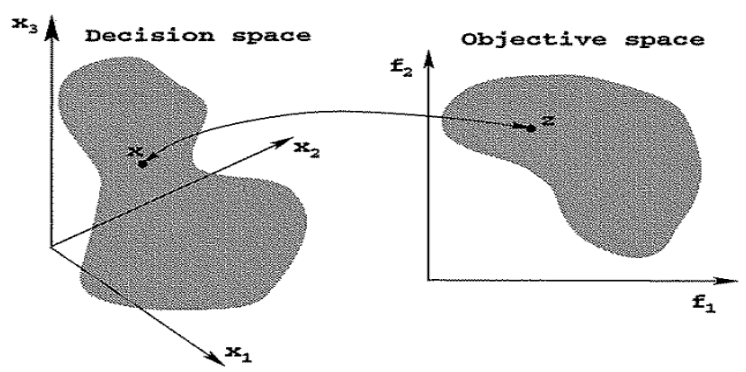

Figure 1. Representation of the decision variable space and corresponding objective space (three decision variables and two objective functions) [12].

There are no irrevocable decisions throughout the process, and the method is not too demanding regarding the information required from the DM. A flowchart of the general framework of the interactive method is depicted in Fig. 2.

Graphical and numerical information are provided and updated at each iteration. Assuming three objectives, the graphical information in the $3 \mathrm{D}$ objective space, with each axis corresponding to an objective, is:

- The range of values allowed for each objective function $(\mathrm{OF})$, using the ideal and nadir points

- The currently known non-dominated solutions

- A color hierarchy regarding the different regions, otherwise explored

- The sub-region which the DM wants to explore (named region of interest).

The numerical information provided is the following:

- Value of the efficient solutions (decision space)

- Value of non-dominated solutions (objective space). 


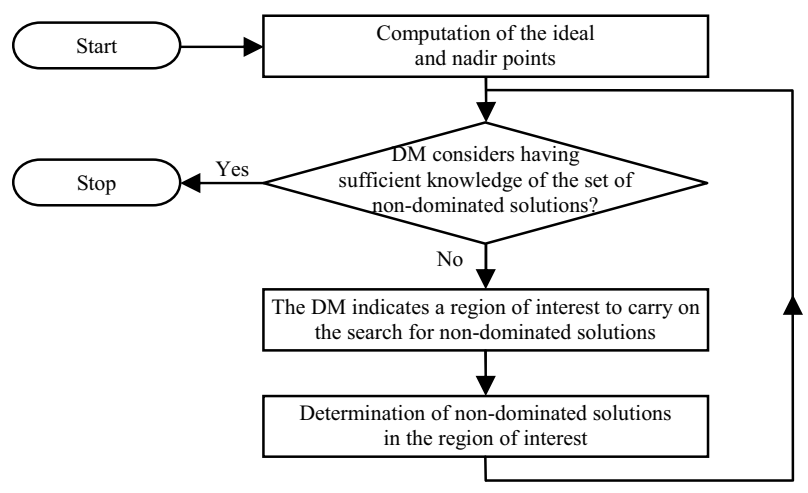

Figure 2. Flowchart of the general framework of the interactive method.

The method starts out by computing the ideal and nadir points (respectively, the best and worst objective values of the solutions in the complete Pareto set). Using both points the initial region is drawn in the objective space, representing the (estimated) range of values for each OF. The resulting region is a rectangular parallelepiped, which is unexplored with regards to the existence of non-dominated solutions.

Until the DM considers having sufficient knowledge on the set of non-dominated solutions (or all the objective space was explored and all non-dominated solutions therefore obtained), the following iterations are performed.

The DM is required to indicate a sub-region to carry on the search for non-dominated solutions. This can be done by: choosing a pair of (currently) adjacent non-dominated solutions or by imposing bounds on the OFs. In the latter, the bounds can be defined numerically or graphically, and the definition of the sub-region (region of interest) should be done within the non-dominated unexplored region. Using a weighted sum program, the original formulation with the bounds defined by the DM is solved to optimality.

The obtained non-dominated solution allows characterizing regions in the objective space. Unfeasible and dominated regions are assigned the hierarchically higher color, and non-dominated regions are colored afterwards: firstly, with the second hierarchically higher color, regions where found solutions have only one OF better than any one solution obtained thus far; then, with the hierarchically lowest color, regions where found solutions have two OFs better than any currently obtained solution. With this color coding, the DM is easily aware of regions where found solutions provide a tradeoff which (s)he may consider potentially more advantageous.

\section{Visual EXPLORATION TOOL}

The adopted visualizations, detailed as follows, were developed to be integrated into a tool, taking into consideration the target user profile. The tool was developed for Windows platforms using XAML and $\mathrm{C \#}$. The target user will be someone with higher education, having a good knowledge of the problem, some computer literacy, and that may use these methods infrequently.

The application has three main options: "Model", "Explore", and "Solutions". In "Model", the user can create/load/edit/save the formulation of a 3-objective MOMIP problem and start the interactive method, "Solve".
Then, using the GLPK software (a package for solving mixed-integer programming) the three solutions that optimize each $\mathrm{OF}$ are obtained. This is the first step of the interactive method, allowing drawing the 3D object, displayed in "Explore". Moreover, by optimizing each OF separately, the first three solutions are obtained and characterization of the different regions is performed accordingly (Fig. 3).

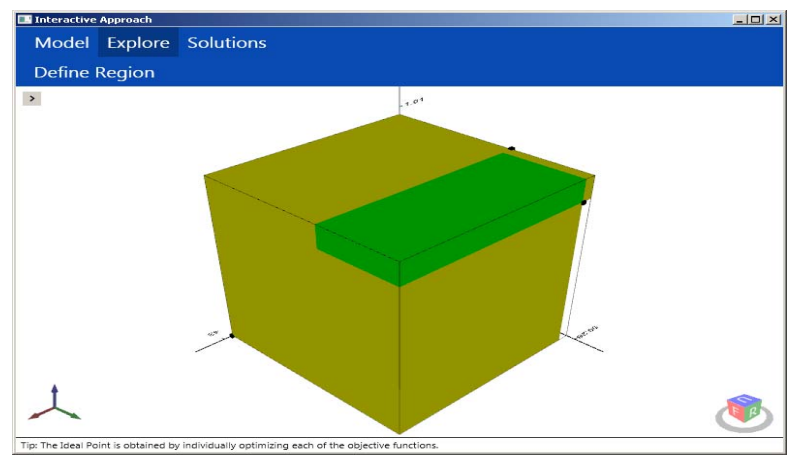

Figure 3. Option "Explore" selected (after obtaining the first three solutions).

The chosen color hierarchy is: red overrides yellow which, in turn, overrides green. Red is for unfeasible/dominated regions, yellow for non-dominated unexplored regions (with only one OF better) and green for non-dominated unexplored regions (with two OFs better). The sub-region which the DM wants to explore (region of interest) is colored blue.

In the "Explore" option the user is able to interact with the representation of the objective space, i.e., the variation allowed for each OF value. Each cube (colored black) represents a solution (corresponding OF values can be read by projecting the cube on each axis). The user may navigate in this 3D environment and search for non-dominated solutions by defining the region of interest (Fig. 4, left).

Once this region is defined, option "Find Solution" becomes available. By pressing it, the model is updated with the defined bounds and solved by the GLPK software.

If a new solution is found, the object is updated to reflect the new region characterization. This is depicted in Fig. 4, right, where the green region (possibly the most interesting one) is reduced and the red region indicates that no solution can be found there (removed in the next iteration). This is to be performed as long as the user considers not having sufficient information of the Pareto set (see Fig. 5 for the region characterization after successive searches for solutions).

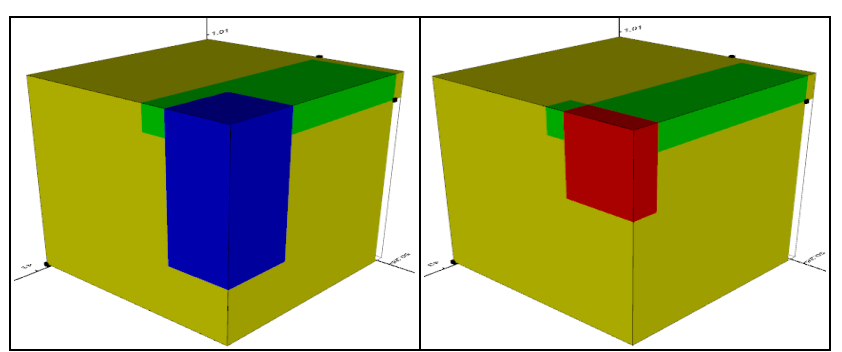

Figure 4. "Explore" option with user-defined region of interest colored blue (left) and region characterization after obtaining a new solution (right). 


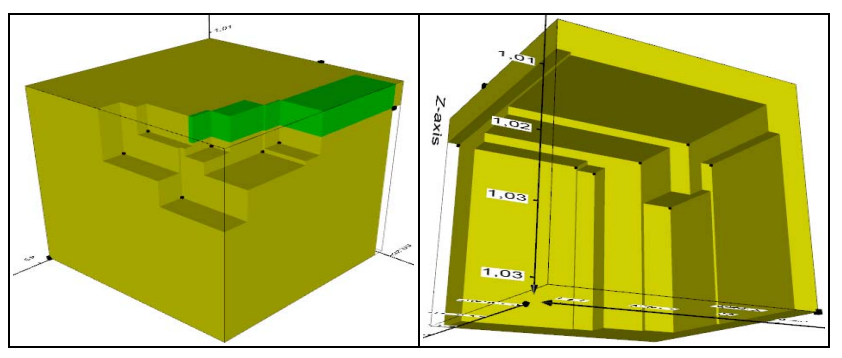

Figure 5. Region characterization after several iterations obtaining solutions, front-view (left) and rear-view (right).

Although information concerning the OF values is very important for DMs, often it is not enough. Therefore, decision space information is crucial for correctly supporting decisions. In the proposed visualization (objective space) the user is able to access this information (decision and slack variables values) and update the graphical representation of the solution.

The "Explore" option therefore allows the user to search for more solutions, and have a first view of their data and graphical representation. The user can freely navigate in the 3D environment, i.e., rotate, zoom in/out, look into/from within, etc. (Fig. 5, right). To further assist the search for interesting solutions, a left expandable panel with several functionalities is available (Fig. 6) with the ability to use transparency, center on a specific solution and insert a solution numerically.

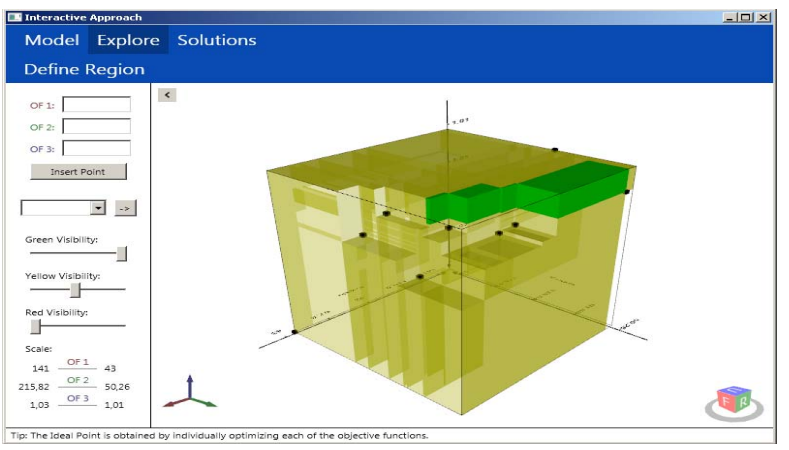

Figure 6. Left panel displayed, red regions not visible, and yellow regions at $50 \%$ transparency.

Finally, in "Solutions", currently obtained solutions can be compared. To that end, "Solution Data" allows seeing full solution data; and "Compare Solutions" allows overlapping solution's graphical representations (with the ability to change the offset and transparency, Fig. 7).

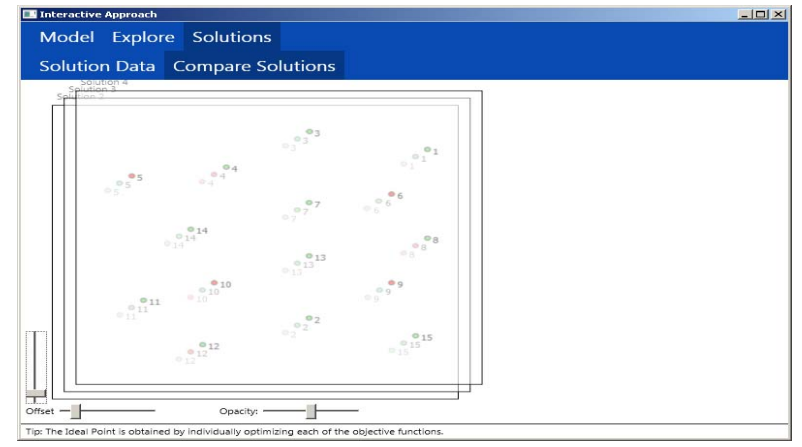

Figure 7. Tool with option "Solutions" > "Compare Solutions" selected.

\section{PRELIMINARY USABILITY EVALUATION}

To evaluate the proposed visualizations, usability tests were performed with the collaboration of 9 users. These were Computer Engineering students attending an introductory course on Human-Computer Interaction, partially fitting the user profile (not having knowledge of the problem addressed), however, familiar with usability principles and guidelines.

Observation techniques and questionnaires [13][14] were used. Users performed a set of 10 tasks regarded representative of operations to be performed by target users - in this case, concerning determining the location of undesirable facilities.

Users had to complete each task in a given time window, and data concerning their performance was collected by an observer. The tasks can be grouped according to their goal:

- Loading data into the tool (the mathematical model and solutions graphical representation)

- Obtaining data from different visualizations

- Interacting in order to obtain new solutions.

The first group of tasks (tasks 1, 3 and 9) aims at evaluating some interface features (e.g. using menu and toolbar options) and ability to input data. The following (tasks 2, 4, 6, 7 and 10) were directed at evaluating easiness of reading data from the different visualization options. The last group of tasks (5 and 8) intended to test ease of use of interacting with the tool in order to obtain new solutions, thus also required understanding displayed data. For each user performing each task, the following data was collected:

- Time required to perform the task

- If the task was completed successfully

- If mistakes were made

- If the user felt lost

- Whether help was requested from the observer

- Difficulty to complete the tasks (both judged by the user and the observer).

After completion of the tasks, users were asked about age, gender, and previous experience with 3D applications; and to evaluate several features using a 5-point Likert-like scale. The questionnaire results, as well as results concerning the list of tested features can be seen in Table I.

Although an overall positive opinion can be inferred, concerns were raised regarding ease of use, consistency in the displayed information, and the need to have further experience in either the tool or the problem addressed.

TABLE I. USERS' OPINION ON GENERAL AND SPECIFIC ASPECTS OF THE TOOL (SCALE: 1 - COMPLETE DISAGREEMENT TO 5 - COMPLETE AGREEMENT)

\begin{tabular}{|l|c|}
\hline \multicolumn{1}{|c|}{ Feature } & Median \\
\hline Is easy to learn & 4.0 \\
\hline Organization is understandable & 3.0 \\
\hline Response time is reasonable & 4.0 \\
\hline Is easy to use & 3.0 \\
\hline Information layout is adequate & 4.0 \\
\hline Help is needed using some functionalities & 4.0 \\
\hline Further knowledge or tool usage experience is required & 3.0 \\
\hline Text is easy to read & 5.0 \\
\hline Relevant information is emphasised & 4.0 \\
\hline Amount of visible information is adequate & 4.0 \\
\hline Is easy to navigate & 4.0 \\
\hline
\end{tabular}


Data obtained from user performance in each of the tasks (Table II) and the dot plots of the time taken to perform them (Fig. 8), suggests that the most difficult tasks were related with interacting with the tool to obtain new solutions (tasks 5 and 8), and reading some specific data (tasks 4, 6, 7 and 10).

TABLE II. USER PERFORMANCE IN EACH TASK (CORRECT COMPLETENESS, WITH ERRORS UNTIL COMPLETENESS, USERS WHO FELT LOST OR REQUESTED HELP, AND MEDIAN VALUES FOR EASINESS FELT AND OBSERVED)

\begin{tabular}{|c|c|c|c|c|c|c|}
\hline Task & $\begin{array}{c}\text { Corr. } \\
\text { comp. }\end{array}$ & $\begin{array}{c}\text { With } \\
\text { errors }\end{array}$ & Felt lost & $\begin{array}{c}\text { Req. } \\
\text { help }\end{array}$ & Easi. felt & $\begin{array}{c}\text { Easi. } \\
\text { obs. }\end{array}$ \\
\hline $\mathbf{1}$ & 9 & 1 & 0 & 0 & 5.0 & 5.0 \\
\hline $\mathbf{2}$ & 9 & 2 & 3 & 1 & 4.0 & 5.0 \\
\hline $\mathbf{3}$ & 9 & 0 & 0 & 0 & 5.0 & 5.0 \\
\hline $\mathbf{4}$ & 8 & 4 & 5 & 1 & 3.0 & 3.0 \\
\hline $\mathbf{5}$ & 8 & 5 & 6 & 2 & 4.0 & 3.0 \\
\hline $\mathbf{6}$ & 9 & 6 & 6 & 2 & 3.0 & 3.0 \\
\hline $\mathbf{7}$ & 9 & 3 & 6 & 1 & 4.0 & 4.0 \\
\hline $\mathbf{8}$ & 8 & 8 & 8 & 4 & 3.0 & 2.0 \\
\hline $\mathbf{9}$ & 9 & 0 & 1 & 0 & 5.0 & 5.0 \\
\hline $\mathbf{1 0}$ & 9 & 5 & 5 & 3 & 3.5 & 3.0 \\
\hline
\end{tabular}

In task 8 users had to navigate in the $3 \mathrm{D}$ object and find solutions with better results concerning one OF. Errors were mostly due to users' inability to correctly identify the subregion to search. When the sub-region was clearly identified using colors (task 5) fewer errors occurred. Also concerning the $3 \mathrm{D}$ object users experienced difficulty in finding some information (tasks 6 and 7). Identifying data in the solution representation was also considered error prone (tasks 4 and 10); although this aspect was not specific of the proposed tool. The remaining tasks were considered trivial.

Results, although generally positive concerning ease of learning, ease of use and satisfaction, suggest some specific visualization solutions may need further improvement.

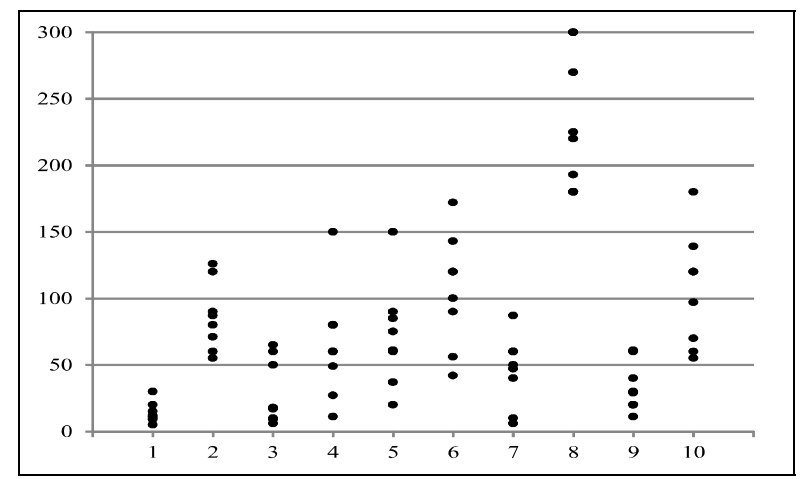

Figure 8. Dot plots of time spent in each of the ten tasks (in seconds).

\section{CONCLUSIONS}

This paper presents information visualization and user interaction issues, relevant to decisions concerning several conflicting objectives. When multi-objective optimization models are used, a large number of non-dominated solutions may exist which have to be obtained and analyzed, becoming a difficult task for DMs. The profile of target users (DMs) as well as the tasks they have to perform was taken into consideration in the development of the proposed visualization and interaction solutions. Usability evaluation was performed which allowed finding usability problems and gather new ideas, helping improving the proposal.

As several scenarios may fit the use of MOMIP, the proposed visualization solutions are left intentionally broad, requiring further development when a specific problem is faced (e.g., in finding the best location for a facility, where interacting with geographic information systems may be important).

As future work, testing the proposed visualizations using virtual reality and different input devices may be promising research avenues. Also, testing the tool in real-life decision scenarios may provide further information concerning its applicability and future improvements.

\section{ACKNOWLEDGMENTS}

The authors are grateful to the students that participated in the evaluation. Work supported by Portuguese funds through the CIDMA - Center for Research and Development in Mathematics and Applications, and the Portuguese Foundation for Science and Technology ("FCT - Fundação para a Ciência e a Tecnologia"), within project PEst-OE/MAT/UI4106/2014.

\section{REFERENCES}

[1] D. Bertsimas, C. Darnell, and R. Soucy, "Portfolio Construction Through Mixed-Integer Programming at Grantham, Mayo, Van Otterloo and Company", Interfaces, vol. 29, 1999, pp. 49-66.

[2] Y. Pochet and L. A. Wolsey, Production Planning by Mixed Integer Programming, Springer, 2006.

[3] M. Goetschalckxa, C. J. Vidal, and K. Dogan, "Modeling and design of global logistics systems: A review of integrated strategic and tactical models and design algorithms", European Journal of Operational Research, vol. 143, 2002, pp. 1-18.

[4] M. J. Alves and J. Clímaco, "An interactive reference point approach for multiobjective mixed-integer programming using branch-and-bound", European Journal of Operational Research, vol. 124, 2000, pp. 478-494.

[5] M. Caramia and P. Dell'Olmo, Multi-objective Management in Freight Logistics, Springer, 2008.

[6] K. Miettinen, F. Ruiz, and A. P. Wierzbicki, "Introduction to multiobjective optimization: interactive approaches", in J. Branke, K. Deb, K. Miettinen, and R. Słowiński (Eds.), Multiobjective Optimization: Interactive and Evolutionary Approaches, Springer, 2008, pp. 27-57.

[7] S. Sayin, "Multi-objective optimization and decision support systems", in C. A. Floudas and P. M. Pardalos (Eds.), Encyclopedia of Optimization, 2nd ed., Springer, 2009, pp. 2460-2465.

[8] M. J. Alves and J. Clímaco, "A review of interactive methods for multiobjective integer and mixed-integer programming", European Journal of Operational Research, vol. 180, 2007, pp. 99-115.

[9] M. J. Alves and J. Clímaco, "Multi-objective mixed integer programming", in C. A. Floudas and P. M. Pardalos (Eds.), Encyclopedia of Optimization, 2nd ed., Springer, 2009, pp. 2454-2460.

[10] R. B. Lopes, Location-Routing Problems of Semi-Obnoxious Facilities: Approaches and Decision Support, PhD thesis, University of Aveiro, 2011

[11] C. Ferreira, B. S. Santos, M. E. Captivo, J. Clímaco, and C. C. Silva, "Multiobjective location of unwelcome or central facilities involving environmental aspects - a prototype of a decision support system", Belgian Journal of Operations Research, Statistics and Computer Science, vol. 36, 1996, pp. 159-172.

[12] K. Deb, Multi-Objective Optimization using Evolutionary Algorithms, John Wiley \& Sons, 2001

[13] C. Ware, Information Visualization - Perception for Design, Morgan Kaufmann, 2000.

[14] P. P. Mitchell, A Step-by-step Guide to Usability Testing, iUniverse, 2007. 